\begin{abstract}
Background: In aromatherapy, essential oils are used as anti-inflammatory remedies, but experimental studies on their action mechanisms are very limited. Aims of the study: To assess their anti-inflammatory activities, the effects of essential oils on neutrophil recruitment in mice were examined in vivo.

Method: The effect of essential oils on leukocyte and neutrophil recruitment induced $6 \mathrm{~h}$ after intraperitoneal injection of casein in mice was examined.

Results: Leukocyte recruitment into the peritoneal cavity in mice was suppressed by intraperitoneal injections of geranium, lemongrass and spearmint oils at the dose of $5 \mu 1 /$ mouse, but was not by tea tree oil. This recruitment was inhibited dose-dependently by geranium oil. The suppression of leukocyte recruitment resulted from inhibition of neutrophil accumulation.

Conclusion: Some essential oils used as anti-inflammatory remedies suppress neutrophil recruitment into the peritoneal cavity in mice.
\end{abstract}

Key words: Inflammation, Perargonium asperum, Melaleuca alternifolia

\section{Suppression of neutrophil recruitment in mice by geranium essential oil}

\author{
Shigeru Abe ${ }^{1, C A}$, Naho Maruyama ${ }^{1}$, \\ Kazumi Hayama, ${ }^{1,2}$, Shigeharu Inouye', \\ Haruyuki Oshima ${ }^{2}$ and Hideyo Yamaguchi ${ }^{1}$
}

${ }^{1}$ Teikyo University Institute of Medical Mycology, 359 Otsuka, Hachioji, Tokyo 192-0395, Japan and ${ }^{2}$ Department of Bioengineering, Faculty of Technology, Teikyo University, 1-1, Toyosato-dai, Utsunomiya, Tochigi 320-0003, Japan

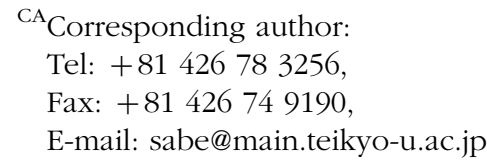

activity against neutrophil adherence in vitro, on casein-induced neutrophil recruitment into the peritoneal cavity of mice.

Essential oils are a folk medicine and recently their use has expanded worldwide to include therapy against various kinds of inflammatory diseases, such as allergy, rheumatism, arthritis and bronchitis. These activities have mainly been recognized through clinical experience, but have been little elucidated experimentally.

Recently several investigators suggested that tea tree $^{1,2}$ and lavender oils ${ }^{3}$ suppressed allergic symptoms through the suppression of histamine release $\mathrm{e}^{4-6}$ and cytokine production. ${ }^{7}$ However, the antiinflammatory activity of various types of essential oils and the cellular mechanisms underlying these actions remain to be clarified. The effects of essential oils on neutrophil function in particular have not been investigated, although the neutrophils are well recognized to play a major regulatory role in inflammation.

We were interested in assessing the modulatory activity on the inflammatory reaction of the neutrophils by the oils. In a preceding paper, ${ }^{8}$ we reported that the essential oils lemongrass, geranium and spearmint suppressed the adherence response of neutrophils in vitro. On the other hand, lavender and tea tree oils, which are traditionally used for inflammatory symptoms, did not show strong activity. In the present study, we investigated the in vivo effects of the essential oils that showed suppressive

\section{Materials and methods}

\section{Essential oils}

The essential oils used are presented in Table 1 with their sources and main constituents. Table 1 also indicates literature references that show clinical use related to inflammatory symptoms. Essential oils were purchased from Hyperplants, Ltd. (Tokyo, Japan).

Each essential oil was diluted to $2.5 \%, 5 \%$, and $10 \%$ solution by $2.5 \%$ dimethyl sulfoxide (DMSO) and 50 $\mu \mathrm{l}$ of Tween 20 was added to $4 \mathrm{ml}$ of the essential oil solution.

\section{Agents}

Polyoxyethylene (20) sorbitan monolaurate (Tween 20) was purchased from Wako Pure Chemical Industries, Ltd. (Osaka, Japan). Casein sodium was purchased from Tokyo Kasei Kogyo Co., Ltd. (Tokyo, Japan), dissolved into 8\% solution and autoclaved ( $121^{\circ} \mathrm{C}$ for $15 \mathrm{~min}$ ). Phosphate-buffered saline (PBS) was purchased from Invitrogen Corp. (Carlsbad, CA, USA) and stored at $4^{\circ} \mathrm{C}$. Diff-Quik was purchased from International Reagents Corp. (Hyogo, Japan). 
Table 1. Essential oils, their main constituents, their sources and manufacturers

\begin{tabular}{lcllc}
\hline Essential oil & Latin name parent plant & Main constituents & Source & References \\
\hline Geranium Bourbon & Perargonium asperum & Geraniol, beta-citronellol & Sanoflore (France) & $9,11,12$ \\
Lemongrass & Cymbopogon citratus & Citral & Sanoflore (France) & 11,12 \\
Spearmint & Mentha spicata & Carvone & Sanoflore (France) & 11,12 \\
Tea tree & Melaleuca alternifolia & Terpinen-4-ol & Sanoflore (France) & 11,12 \\
\hline
\end{tabular}

\section{Animals}

All animal experiments were performed according to the guidelines for the care and use of animals approved by Teikyo University. Six-week-old female ICR mice (Charles River Japan, Inc., Yokohama, Kanagawa, Japan) were used for all animal experiments. The photoperiods were adjusted to $12 \mathrm{~h}$ of light and $12 \mathrm{~h}$ of darkness daily, and the environmental temperature was constantly maintained at $21^{\circ} \mathrm{C}$. The mice were kept in cages housing five or six animals and were given ad libitum access to food and water.

\section{Neutrophil recruitment assay}

Three milliliters of $8 \%$ casein sodium solution was injected intraperitoneally to mice. Both 2 and $4 \mathrm{~h}$ later, $0.2 \mathrm{ml}$ of the essential oil solution was injected. A dose of $2.5 \%$ solution corresponds to $5 \mu \mathrm{l}$ of pure oil. Control mice received $0.2 \mathrm{ml}$ of $2.5 \%$ DMSO solution. Mice were sacrificed by carbon dioxide $6 \mathrm{~h}$ after casein injection.

Three milliliters of PBS was injected intraperitoneally to the killed mice, and $2 \mathrm{ml}$ of solution was taken from the peritoneal cavity to collect leukocytes. After centrifugation at $350 \times \mathrm{g}$ at $4^{\circ} \mathrm{C}$ for $5 \mathrm{~min}$, the precipitate was suspended in $2 \mathrm{ml}$ of saline. The cell number of leukocytes was measured by Celltac (Nihon Kohden Corporation, Tokyo, Japan). The cell number of neutrophils recovered from the peritoneal cavity was measured by cytocentrifigation and DiffQuik staining as described previously. ${ }^{9}$ Briefly, $20 \mu \mathrm{l}$ of leukocyte suspension was added to $180 \mu \mathrm{l}$ of PBS containing 10\% heat-inactivated fetal calf serum, then poured into a plastic tube with a slide glass and cytocentrifuged at $75 \times \mathrm{g}$ for $5 \mathrm{~min}$, and the slide glass was then stained by Diff-Quik. The percentage ratio and the cell number of neutrophils were calculated by counting the neutrophil number of more than 50 leukocytes/sample. The relative numbers of leukocytes and neutrophils recruited into the peritoneal cavity were expressed by the ratio to those in $2.5 \%$ DMSO solution without oils.

\section{Statistical analysis}

The data were compared using the non-parametric Mann-Whitney U-test.

\section{Results}

Effects of essential oils on casein-induced leukocyte recruitment into the peritoneal cavity of mice

About $4 \times 10^{6}$ calls of leukocytes were recovered by washing from the peritoneal cavity of normal mice and $(2 \sim 3) \times 10^{7}$ cells from the cavity of mice injected intraperitoneally with $3 \mathrm{ml}$ of casein solution. As shown in Fig. 1, $5 \mu \mathrm{l}$ of lemongrass, spearmint and geranium oil significantly lowered the infiltration of leukocytes to $51.8 \%, 62.5 \%$ and $70.2 \%$ compared with control (100\%), respectively, while the administration of $5 \mu \mathrm{l}$ of tea tree oil had no influence on the leukocyte infiltration (102.4\%).

\section{Effects of geranium oil on leukocyte recruitment into the peritoneal cavity of mice}

Although lemongrass oil was most active, it is known to irritate mucosal tissues on contact, ${ }^{10}$ so we further examined the activity of various doses of geranium oil. The mice receiving $20 \mu \mathrm{l}$ of geranium oil exhibited an unusual behavior (sedated condition with loss of normal movement) after the first injection, so a second injection was not made. The results are shown in Fig. 2. All doses of geranium oil in the range between 5 and $20 \mu \mathrm{l} /$ mouse suppressed leukocyte recruitment in a dose-dependent manner, with $10 \mu \mathrm{l}$ of this oil showing the strongest activity (13.8\%).

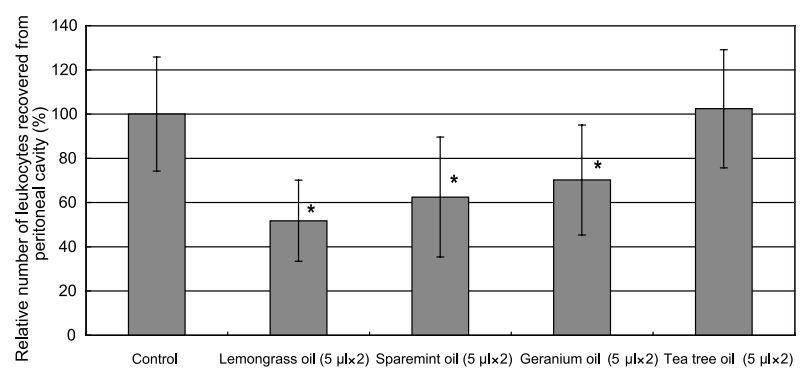

FIG. 1. Effect of essential oils on casein-induced leukocyte recruitment. Essential oils were injected into mice 2 and $4 \mathrm{~h}$ after casein induction. After $6 \mathrm{~h}$, leukocytes in the peritoneal cavity were collected and their numbers were counted. Each value represents an average of four to eight samples and the standard deviation. ${ }^{*} p<0.05$. 


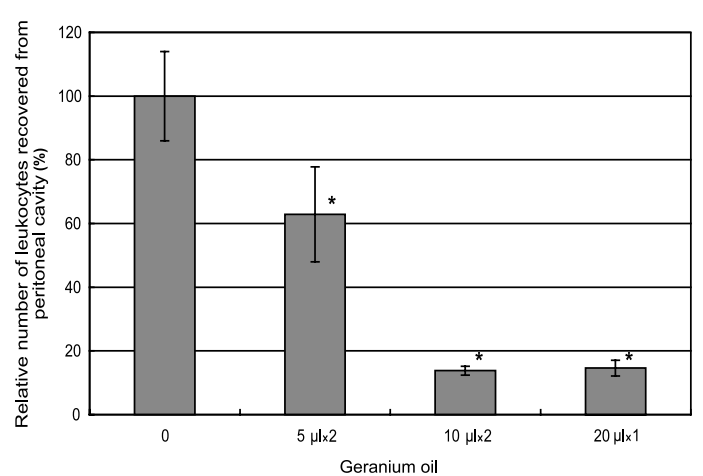

FIG. 2. Effect of geranium oil on casein-induced leukocyte infiltration. Geranium oil was injected 2 and $4 \mathrm{~h}$ after casein induction. After $6 \mathrm{~h}$, peritoneal leukocytes were counted. Each value represents an average of four to six samples and the standard deviation. ${ }^{*} p<0.05$.
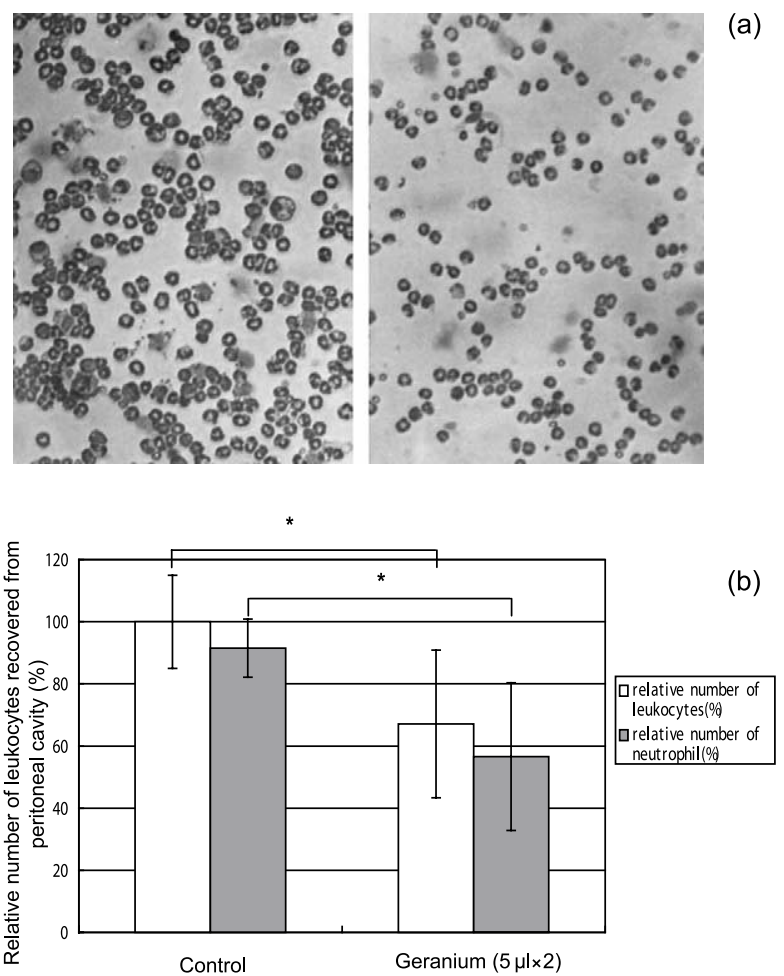

FIG. 3. Effect of geranium oil on casein-induced neutrophil infiltration. Geranium oil was injected 2 and $4 \mathrm{~h}$ after casein induction. After $6 \mathrm{~h}$, peritoneal leukocytes were counted. The neutrophil number was calculated from their content in leukocyte preparation, determined by cytocentrifigation and Diff-Quik staining. (A) Photograph of casein-induced neutrophil infiltration after Diff-Quik staining. (B) The neutrophil number. Each value represents an average of four samples and the standard deviation. ${ }^{*} p<0.05$.

The effect of geranium oil on the ratio of neutrophils in leukocytes recruited by casein injection into the peritoneal cavity was determined using Diff-Quik staining as shown in Fig. 3A. Neutrophils were the major part of casein-induced leukocytes of control and geranium-treated mice: $85-90 \%$ of the suspension taken from the peritoneal cavity was found to be neutrophils. In this experiment, $5 \mu \mathrm{l}$ of geranium oil lowered the number of leukocytes in the peritoneal cavity; neutrophil infiltration was also significantly lowered by geranium oil (Fig. 3B).

\section{Discussion}

In the present study, we showed that intraperitoneal injection of geranium, spearmint and lemongrass oils suppressed leukocyte accumulation induced by casein injection in mice, and geranium oil at the dose of $5 \mu \mathrm{l} /$ mouse especially suppressed the neutrophil recruitment into the peritoneal cavity. The inhibition of leukocyte recruitment by this oil was dose dependent, but tea tree oil did not inhibit leukocyte accumulation. As far as we know, this is the first report indicating that essential oils inhibit neutrophil accumulation in vivo.

In the peritoneal cavity of normal mice, very few neutrophils are usually observed, but $6 \mathrm{~h}$ after casein injection, about $(2 \sim 3) \times 10^{7}$ cells/mouse of neutrophils were recovered. This means that casein injection causes severe peritonitis with neutrophil accumulation perhaps through production of chemotactic peptides. ${ }^{11}$ Therefore, our results suggest that geranium, lemongrass and spearmint oils may alleviate the inflammatory leukocyte response in the peritoneal cavity. This speculation is supported by the finding that the essential oils with suppressive activity for leukocyte accumulation (i.e., lemongrass, geranium and spearmint oils) have been reported to inhibit tumor necrosis factor-alpha-induced neutrophil adherence at very low concentrations $(0.00625 \%)$ in vitro, but tea tree oil without the suppressive activity already described is known to have only limited influence on neutrophil adherence response in vitro. ${ }^{8}$

Brand et al. reported that tea tree oil inhibited histamine-induced edema ${ }^{4}$ but did not change leukocyte infiltration in a murine contact dermatitis model. ${ }^{1}$ On the other hand, Kawabata et al. postulate that application of geranium oils to chronic vaginal candidiasis in combination with tea tree and ravansara oils provides a better therapeutic benefit. ${ }^{12}$ This possible therapeutic activity may reflect the antiinflammatory action of geranium oil through suppression of neutrophil infiltration. In this connection, we must check the possibility that suppression of neutrophil infiltration in vivo by an essential oil may result in a lowering of host defense against Candida infection.

In aromatherapy, several essential oils can be used as therapeutic treatments for inflammatory symptoms with lesional neutrophil accumulation, such as aphthous stomatitis, lesional bacterial or fungal infections. The results described suggest that geranium, lemongrass and spearmint oils may suppress these inflammatory symptoms. We have developed 
murine models of oral ${ }^{13}$ and vulvovaginal candidiasis (manuscript in preparation) showing inflammatory symptoms. Using these models, we wish to investigate the therapeutic benefit of these essential oils and the roles of anti-inflammatory activity in their therapeutic actions.

ACKNOWLEDGEMENTS. This work was supported in part by a grant from the Kampo Medicine Research Fund (Tokyo) and a grant (No.15590401) from the Ministry of Education. Culture, Sports, Science and Technology of Japan.

\section{References}

1. Brand C, Grimbaldeston MA, Gamble JR, Finlay-Jones JJ, Hart PH. Tea tree oil reduces the swelling associated with the efferent phase of a contact hypersensitivity response. Inflamm Res 2002; 51: 236-244.

2. Hart PH, Brand C, Carson CF, Riley Tv, Prager RH, Finlay-Jones JJ Terpinen-4-ol, th e main component of the essential oil of Melaleuca alternifolia (tea tree oil), suppresses inflammatory mediator production by activated human monocytes. Inflamm Res 2000; 49: 619-626.

3. Kim H-M, Cho S-H. Lavender oil inhibits immediate-type allergic reaction in mice and rats. J Pharm Pharmacol 1999; 51: 221-226.

4. Brand C, Townley SL, Finlay-Jones JJ, Hart PH. Tea tree oil reduces histamine-induced oedema in murine ears. Inflamm Res 2002; 51: $283-$ 289.
5. Santos FA, Rao VSN. Mast cell involvement in the rat paw oedema response to 1,8-cineole, the main constituent of eucalyptus and rosemary oils. Eur J Pharmacol 1997; 331: 253-258.

6. Koh KJ, Pearce AL, Marshman G, Finaly-Jones JJ, Hart PH. Tea tree oil reduces histamine-induced skin inflammation. Br J Dermatol 2002; 147: $1212-1217$

7. Brand C, Ferrante A, Prager RH, et al. The water-soluble components of the essential oil of Melaleuca alternifolia (tea tree oil) suppress the production of superoxide by human monocytes, but not neutrophils, activated in vitro. Inflamm Res 2001; 50: 213-219.

8. Abe $\mathrm{S}$, Maruyama $\mathrm{N}$, Hayama $\mathrm{K}$, Ishibashi $\mathrm{H}$, Inoue $\mathrm{H}$, Oshima $\mathrm{H}$, Yamaguchi H. Suppression of TNF-alpha induced neutrophil adherence response by essential oils, Mediat Inflamm 2003; 12: 323-328.

9. Morikawa K, Kikuchi Y, Abe S, Yamazaki M, Mizuno D. Early cellular responses in the peritoneal cavity of mice to antitumor immunomodulators. Gann 1984; 75: 370-378.

10. Bowles EJ. The Basic Chemistry of Aromatherapeutic Essential Oils, Sydney: Pirie Printers, 2000: 49-51.

11. Suzue T, Matsushima A, Inada Y. Cooperative participation of two peptides from beta-casein in leukocyte chemotaxis. Febs Letter 1976; 69: $133-136$.

12. Kawabata K, Yoshi Y, Tamizu S. Effective aromatherapy (in Japanese). Fujinseikatsusha 2002.

13. Takakura N, Sato Y, Ishibashi $\mathrm{H}$, Oshima $\mathrm{H}$, Uchida K, Yamaguchi $\mathrm{H}$, Abe $\mathrm{S}$. A novel murine model of oral Candidiasis with local symptoms characteristic of oral thrush. Microbiol Immunol 2003; 47: 321-326.

\section{Received 24 October 2003}

Accepted 6 November 2003 


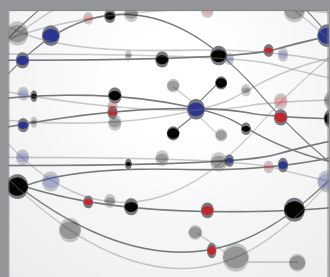

The Scientific World Journal
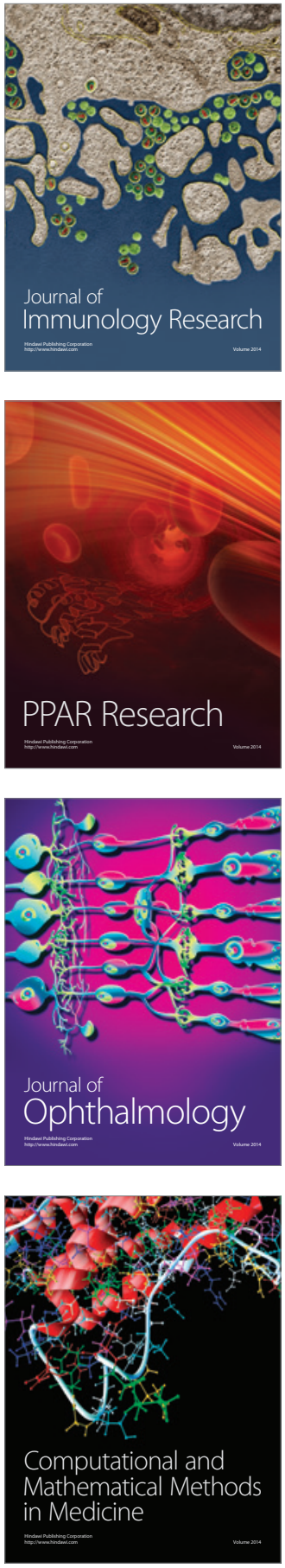

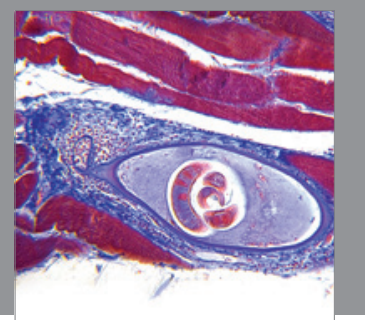

Gastroenterology

Research and Practice
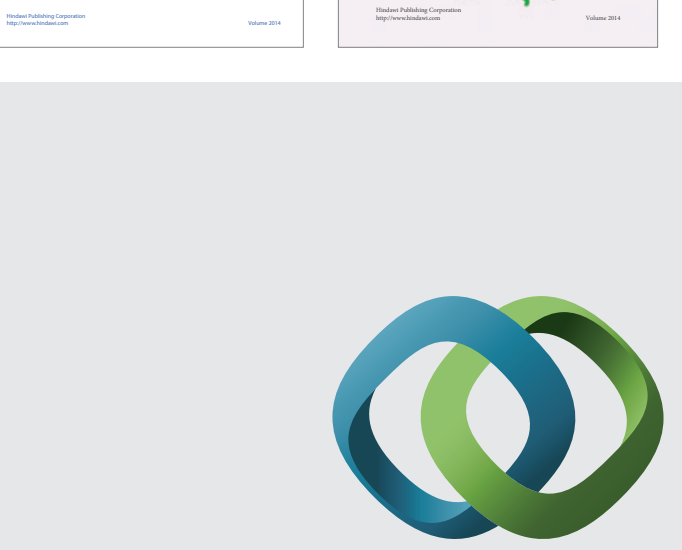

\section{Hindawi}

Submit your manuscripts at

http://www.hindawi.com
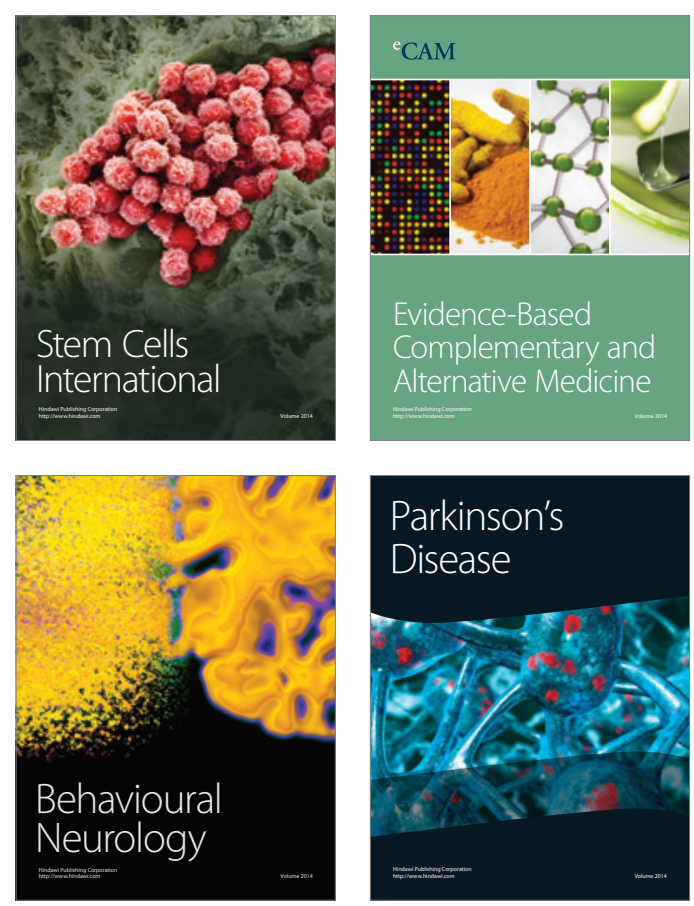

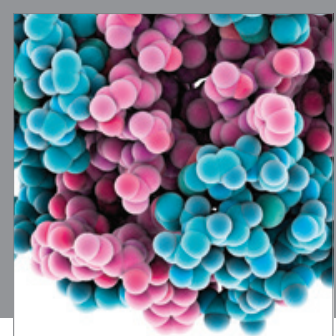

Journal of
Diabetes Research

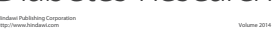

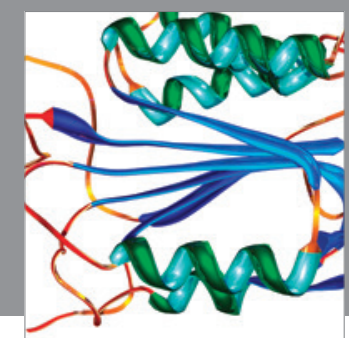

Disease Markers
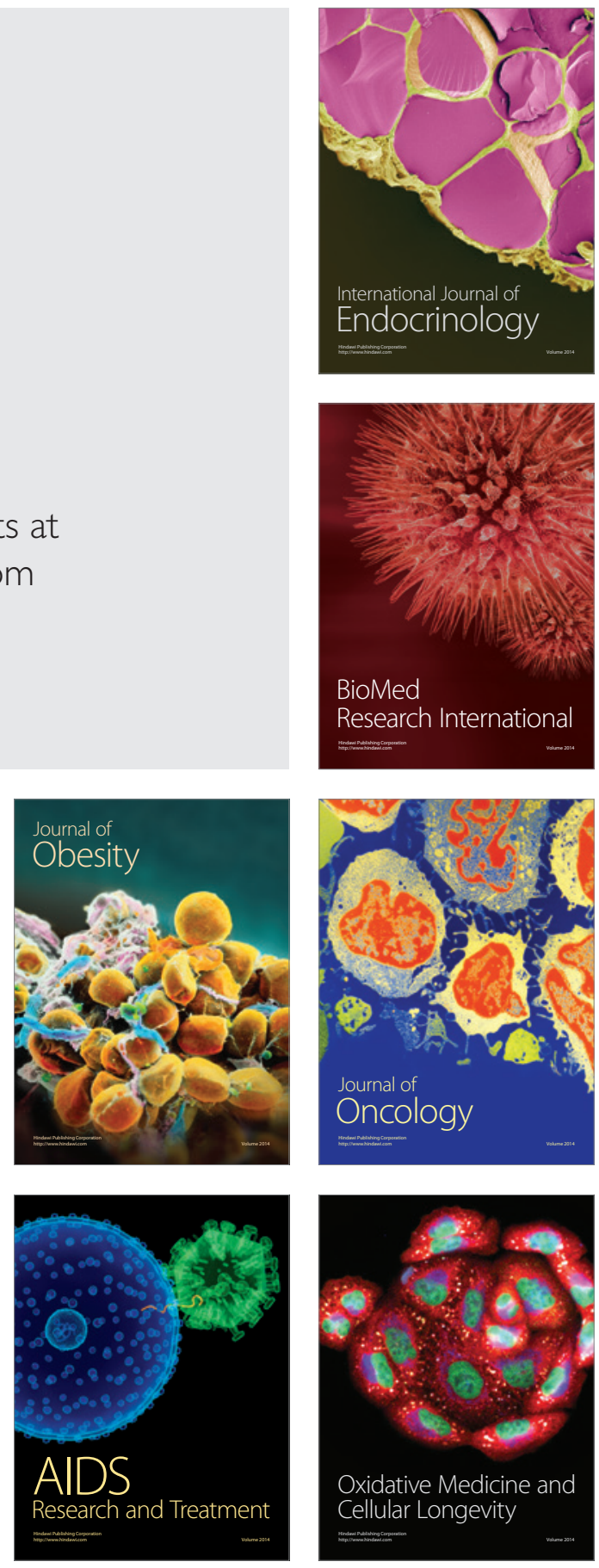